American Journal of Applied Sciences 5 (7): 763-768, 2008

ISSN 1546-9239

(C) 2008 Science Publications

\title{
Short-Term and Medium-Term Load Forecasting for Jordan's Power System
}

\author{
${ }^{1}$ I. Badran, ${ }^{2} \mathrm{H}$. El-Zayyat and ${ }^{2}$ G. Halasa \\ ${ }^{1}$ Electrical Engineering Department, Philadelphia University, Jordan \\ ${ }^{2}$ Electrical Engineering Department, The University of Jordan, Jordan
}

\begin{abstract}
Several electric power companies are now forecasting electric loads based on conventional methods. However, since the relationship between loads and factors influencing these loads is nonlinear, it is difficult to identify its nonlinearity by using conventional methods. Most of papers deal with 24-h-ahead load forecasting or next day peak load forecasting. These methods forecast the demand power by using forecasted temperature as forecast information. But, when the temperature curves change rapidly on the forecast day, loads change greatly and forecast error would be going to increase. Typically, load forecasting can be long-term, medium-term, short-term or very short-term. This paper concentrates on short-term load forecasting and partially on medium-term load forecasting applying regression models.
\end{abstract}

Key words: Load forecasting, $\mathrm{R}^{2}$ value, regression models, least square

\section{INTRODUCTION}

The operation of modern power systems is usually associated with a variety of operations planning procedures. Operations planning encompass methodologies and decision processes by which an electric power system is prepared to meet the electric load within a set of specified technical performance criteria as well as economic performance criteria. The operations planning process must start with a projection of what the electric load will be at future time intervals of interest, i.e. load forecasting. Depending on the application (i.e., near future operations planning, expansion planning, etc.), load forecasting is classified as (a) short range ( $\sim$ day), (b) medium range ( $\sim 1$ week to 1 month) and (c) long range (several years). Each class of load forecasting uses different models to meet the specific objectives of the application. The present work applies the regression load forecasting models, concentrating on short-term and medium-term load forecasting for Jordan power system. The results obtained are necessary for operations planning. Long range load forecasting is also necessary for expansion planning $^{[1]}$.

\section{ELECTRIC LOAD COMPONENTS AND CHARACTERISTICS}

The prediction of the electric load at a future time is a challenging problem because of the diverse characteristics of the electric load and the uncertainty associated with them. A typical daily variation of electric loads is shown in Fig. 1. The characteristics of the electric load depend on the nature of the users and the end use devices utilized such as motors, air conditioners, lighting, etc. From this point of view, the electric load can be separated into four major categories:

- Residential,

- Commercial,

- Agricultural and

- Industrial

\section{ELECTRIC LOAD FORECASTING MODELS}

The basic load forecasting model can be described as follows ${ }^{[2]}$ :

Consider a future time interval $\left(t_{k}, t_{k+1}\right)$. Let the electric load at this time be: $\mathrm{d}(\mathrm{t})$

$$
\mathrm{d}(\mathrm{t}), \mathrm{t}_{\mathrm{k}} \leq \mathrm{t} \leq \mathrm{t}_{\mathrm{k}+1}
$$

And the consumed energy be $\mathrm{E}(\mathrm{k})$

$$
E(k)=\int_{t_{k}}^{t_{k}+1} d(t) d t
$$




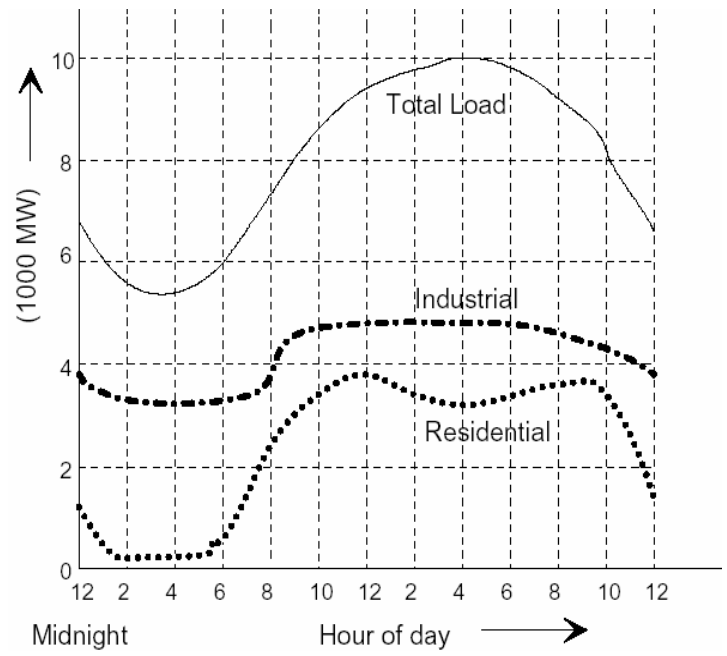

Fig. 1: Typical daily variation of electric loads

Then average electric load is:

$$
d_{a}(k)=\frac{E(k)}{\left(t_{k+1}-t_{k}\right)}
$$

And the peak load is:

$$
d_{p}(k)=\operatorname{Max}_{t=(t k, t k+1)} d(t)
$$

Load forecasting techniques encompass any methodology by which the statistics of $\mathrm{da}_{(\mathrm{k})}$ or $\mathrm{dp}_{(\mathrm{k})}$ can be computed. Many times the forecast of $\mathrm{da}_{(\mathrm{k})}$ is referred to as energy forecast (or average load forecast) and the forecast of $\mathrm{dp}_{(\mathrm{k})}$ is referred to as peak load forecast. The time interval $\left(t_{k}, t_{k+1}\right)$ may be 15 min, an $\mathrm{h}$, a day, a week, or a year depending on the objective of the load forecasting method.

The variables $\mathrm{da}_{(\mathrm{k})}$ and $\mathrm{dp}_{(\mathrm{k})}$ are, in general, considered to be stochastic variables. It is assumed that they obey a certain model which is polluted with noise. Then, the basic problem is to compute the expected values of the stochastic processes $\mathrm{da}(\mathrm{k})$ and $\mathrm{dp}(\mathrm{k})$ and measures of their uncertainty, such as standard deviation, moments, etc. The general load forecasting model is illustrated in Fig. 2. The Fig. 2 shows a typical load forecasting model consisting of three components: (a) the base electric load, (b) the weather sensitive load and (c) the uncertainty model. Note that the net electric load is:

$$
\mathrm{L}(\mathrm{t})=\mathrm{B}(\mathrm{t})+\mathrm{W}(\mathrm{t})+\eta(\mathrm{t})
$$

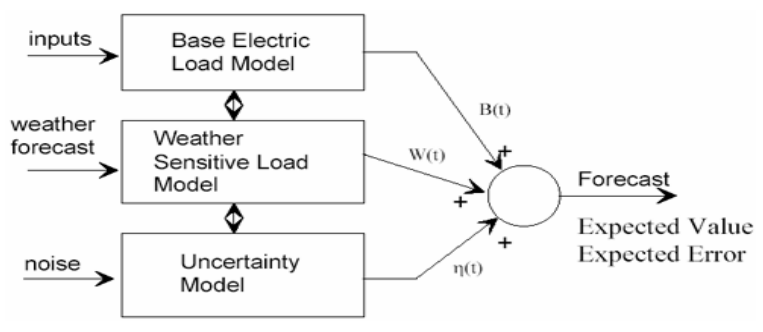

Fig. 2: General load forecasting model

When the time interval is one day or more, it is customary to represent the electric load with the socalled load duration curve.

The load duration curve clearly indicates the peak load. The energy equals the area under the curve. The average load can be computed from the energy by dividing by the time duration $\mathrm{T}$.

\section{PROBABILISTIC MODELS}

The general form of this class of electric load models can be expressed as follows:

$$
\mathrm{d}(\mathrm{k})=\sum_{\mathrm{i}=1}^{\mathrm{n}} \mathrm{aig}_{\mathrm{i}}(\mathrm{k})+\eta(\mathrm{k})
$$

where:

$\mathrm{d}(\mathrm{k})=$ Electric load component to be forecasted (average or peak)

gi $(\mathrm{k})=$ Arbitrarily selected functions

ai $=$ Parameters of the model

$\eta(\mathrm{k})=$ A random variable that describes the random variation of $\mathrm{d}(\mathrm{k})$ about the model

$$
\sum_{i=1}^{n} a_{i} g_{i}(k)
$$

It should be pointed out that the functions gi $\mathrm{i}_{(\mathrm{k})}$ can be selected in different ways. For example, if the functions $g i_{(\mathrm{k})}$ are functions of the time variable $\mathrm{k}$ only, then the model is called a regression model. If the function $\mathrm{gi}_{(\mathrm{k})}$ is expressed in terms of economic parameters, such as population, average income, etc., the resulting model is an econometric model.

\section{FORECASTING MODEL OF WEATHER SENSITIVE ELECTRIC LOADS}

In peak demand forecasting, the weather plays an important role. Actually, for many systems the peak 

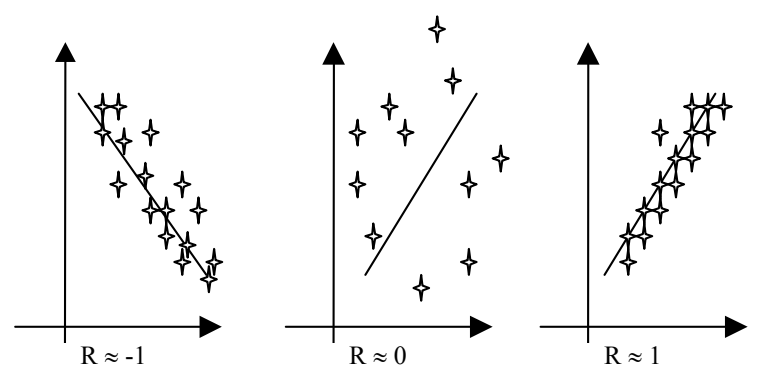

Fig. 3: $\mathrm{R}^{2}$ value curves

demand occurs at times when weather-dependent loads, i.e. heating or air-conditioning are switched-on. The reason for the phenomenon is the high penetration of electric space heaters or air conditioners.

The total system peak demand can be viewed as the sum of a component which is weather insensitive and another component which is weather sensitive, i.e.,

$$
\mathrm{dp}(\mathrm{k})=\mathrm{B}(\mathrm{k})+\mathrm{W}(\mathrm{k})
$$

where:

$\begin{array}{ll}\mathrm{B}(\mathrm{k})= & \begin{array}{l}\text { Weather-insensitive component in time } \\ \text { interval } \mathrm{k}\end{array} \\ \mathrm{W}(\mathrm{k})= & \text { Weather-sensitive component in time interval } \\ & \mathrm{k}\end{array}$

The weather-sensitive component, W (k), represents the electric power demand of weathersensitive devices, such as space heaters and air conditioners. It depends on weather variables such as:

- Coincident dry-bulb temperature, $\mathrm{T}$

- Humidity, h

- Wind velocity, $\mathrm{v}$, etc

Forecasting models for the weather-sensitive component, $\mathrm{W}(\mathrm{k})$, follow the same principles as the models for forecasting the non weather-sensitive component $\mathrm{B}(\mathrm{k})$. Specifically, a model is assumed, the parameters of the model are identified using past data and then the model is used to predict future loads.

To identify the weather-sensitive component, the total demand is sampled (hourly, daily, weekly, annually) together with weather variables. Usually the daily or weekly peaks are recorded. Then model identification procedures can be employed to separate the non weather-sensitive component from the weathersensitive component.

R-squared value: An indicator from 0 to 1 that reveals how closely the estimated values for the trend or regression line correspond with the actual data. A trend line is most reliable when its R-squared value is at or near 1 . This depends on the quality of data and its variability, as shown in Fig. 3. The graphs present the fitted regression lines and contrast them with the actual values for different values of $R$.

The least square method: If an unknown quantity $x$ is measured $n$-times, the measurements $\mathrm{x}_{1}, \mathrm{x}_{2}, \mathrm{x}_{3}, \ldots \ldots, \mathrm{x}_{\mathrm{n}}$ often vary because of uncontrollable factors such as temperature, atmospheric pressure and so forth. Thus, a scientist is often faced with the problem of using $n$ different observed measurements to obtain an estimate $\mathrm{x}^{\prime}$ of an unknown quantity $\mathrm{x}$. One method of making such an estimate is based on the Least Square Principle, which states that, the estimated $x^{\prime}$ should be chosen to $\operatorname{minimize}^{[4]}$ :

$$
\mathrm{S}=\left(\mathrm{x}_{1}-\mathrm{x}^{\prime}\right)^{2}+\left(\mathrm{x}_{2}-\mathrm{x}^{\prime}\right)^{2}+\ldots \ldots \ldots+\left(\mathrm{x}_{\mathrm{n}}-\mathrm{x}^{\prime}\right)^{2}
$$

Econometric approach: For many forecasting purposes econometric models are the most commonly used and most powerful models. They are based on single or multiple regression models. The basic idea of this approach is to use historical annual energy consumption and economic data to derive mathematical equations that explain the relations between electricity demand and other independent variables that influence this demand. If the relation between these variables is significant, then projections could be obtained from the forecast of the independent variables included in the multi-equation econometric model. Econometric approach is used to forecast the electricity sales to domestic, commercial, retail industries and retail water pumping sectors ${ }^{[3]}$.

Regression analysis: The regression analysis performs linear regression analysis by using the "least squares" method to fit a line through a set of observations. You can analyze how a single dependent variable is affected by the values of one or more independent variables. For example, you can analyze how an athlete's performance is affected by such factors as age, height and weight. You can apportion shares in the performance measure to each of these three factors, based on a set of performance data and then use the results to predict the performance of a new, untested athlete. Also we can apply the econometric method by attaching the consumption to the economic and other factors that affect the consumption to apply the linear formula ${ }^{[4]}$ :

$$
\mathrm{Y}=\mathrm{aX} \mathrm{X}_{1}+\mathrm{bX} \mathrm{X}_{2}+\mathrm{cX} \mathrm{X}_{3}+\mathrm{dX}_{4}+\ldots \ldots+\mathrm{fX}_{\mathrm{n}}
$$

where: 
Am. J. Applied Sci., 5 (7): 763-768, 2008

$$
\begin{aligned}
\mathrm{Y} & =\text { Consumption } \\
\mathrm{X}_{1}, \mathrm{X}_{2}, \mathrm{X}_{3}, \mathrm{X}_{4}= & \text { Factors that are affecting the } \\
& \text { consumption } \\
\mathrm{a}, \mathrm{b}, \mathrm{c}, \mathrm{d}, \mathrm{f}= & \begin{array}{l}
\text { Constants that are obtained from the } \\
\text { regression }
\end{array}
\end{aligned}
$$

\section{DATA AND ASSUMPTION}

The data sources ${ }^{[1]}$ used in this forecast are divided into two types:

- Internal data sources

- External data sources

The internal data sources: This category includes each sector energy consumption, maximum demand, load coinciding with system peak, number of consumers...etc, which are obtained from the national and private electric power company sources. These data have a good quality and consistently reported except for a certain data concerning consumers load factors used for computing maximum demands from the energy consumption forecast.

The external data sources: This category includes data such as gross domestic product [GDP], the value added for some sectors, population growth, production...etc. These data levels and population are obtained from various sources, such as the department of statistics, ministry of planning, water authority, central bank and large industries.

\section{LOAD FORECASTING ASSUMPTIONS}

The time of the annual peak load occurrence is predicted on the assumptions that it occurs during daytime (morning peak) instead of the current evening peak. This is due to the fact that morning peak grows higher than the evening peak and the reason for that is firstly the high growth in industrial and commercial sectors and secondly the growth in the percentage of consumers using air-conditioning.

The price of electricity will always be adjusted to remain a true reflection of economic costs. The electricity price has not only affected the energy consumption but also affected the peak demand since the consumers will respond to this price by adopting a suitable mode of operation which secure an efficient utilization of electricity in order to reduce their electricity bill.

Population in Jordan is expected to be 8.16 million inhabitants in the year 2020 with an average annual growth rate of $(2.4) \%$. The return of Jordanian

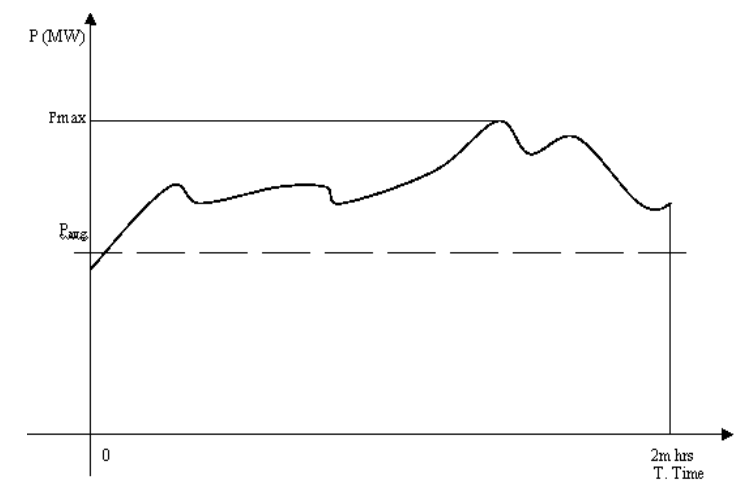

Fig. 4: Daily load curve

expatriates from the gulf, increased the population of Jordan by $10.3 \%$ in 1990 and $6.9 \%$ in 1991. The economy is assumed to grow at an average annual growth rate range of $3.6 \%-5.1 \%$ during the period of this study.

The effect of summer period on the electricity consumption and peak load.

From the experience in the previous summer timing studies, it was shown that the application of summer timing during the period $1^{\text {st }} A$ pril- $1{ }^{\text {st }}$ October, caused a decrease in the electricity consumption and peak load in the range of (20-30) MW. This is due to the absence of heating loads during winter timing. However, it was shown that the increase of one degree centigrade of ambient temperature above the value of $25^{\circ} \mathrm{C}$; caused an increase of (6-8) MW at the peak load.

\section{LOAD CURVE CALCULATIONS}

With a return period of six years, the load curves are calculated based on the following simple equations $^{[1,2]}$ :

$$
\begin{aligned}
\mathrm{L} . \mathrm{F}= & \mathrm{P}_{\mathrm{avg}} / \mathrm{P}_{\max } \\
\mathrm{E}= & \int \mathrm{P} . \mathrm{F} \text { stands for Load Factor. } \\
& \text { Over the period of time (from } 0 \text { to T). } \\
\mathrm{E}= & \mathrm{P}_{\text {avg }} * \mathrm{~T} \\
\mathrm{E}= & \mathrm{P}_{\max } * \mathrm{~L}^{*} \mathrm{~F}^{*} \mathrm{~T} \\
\mathrm{P}_{\max }= & \mathrm{E} /\left(\mathrm{L} . \mathrm{F}^{*} \mathrm{~T}\right)
\end{aligned}
$$

where:

$P$ stands for Power measured in (MW).

E stands for Energy measured in (GWh).

The daily load curve is shown in Fig. 4. 


\section{RESULTS AND DISCUSSIONS}

Computer program results for load forecasting of the Jordan power system are presented in Fig. 5 to 12. The forecast includes the weekends, working days and the two major Muslim religious holidays which are Eid Al-Fiter and Eid Al-Adhaha. Historical data of the daily load curve for the years 1999-2004 are plotted in Figs 5 to 8 for these three types of daily load demands. Based on these data, the medium-term daily load forecasts for the period 2005-2014 are presented in Fig 9-12.

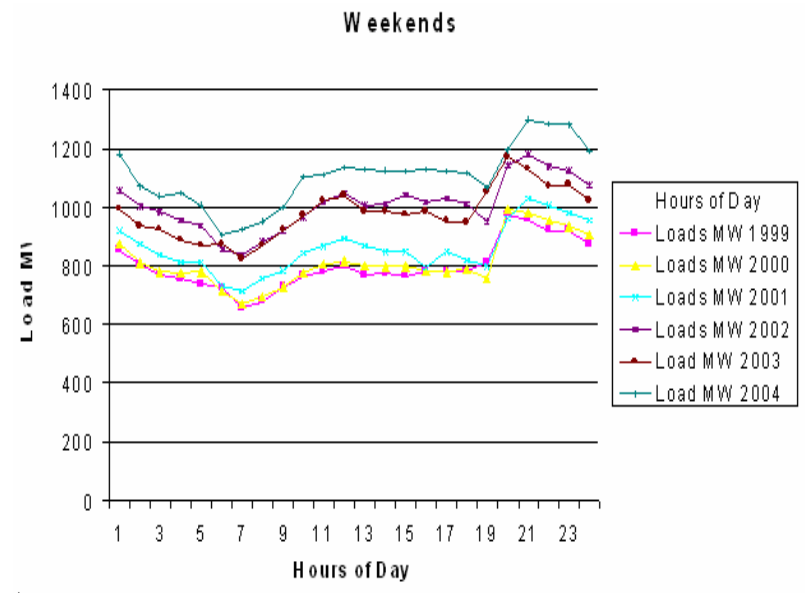

Fig. 5: System consumption at weekends

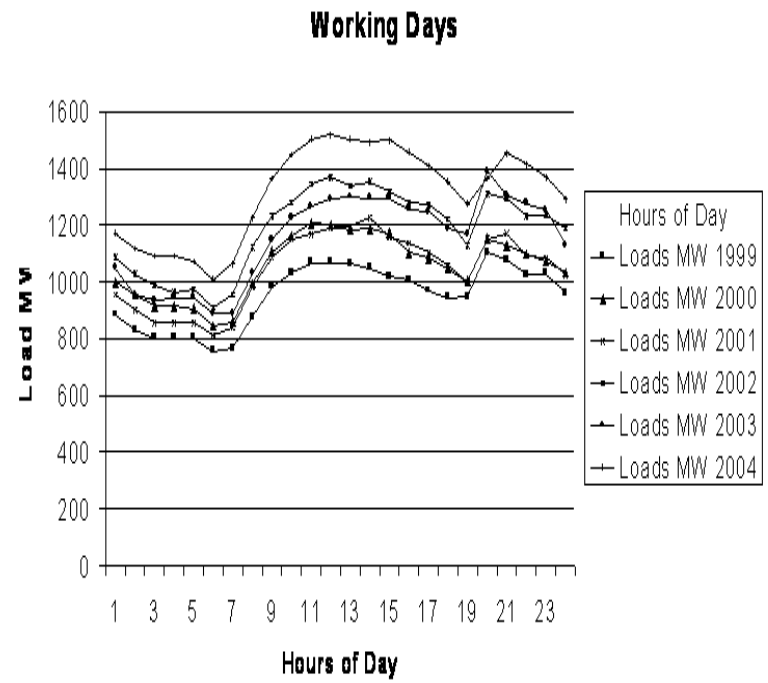

Fig. 6: System consumption on working days.

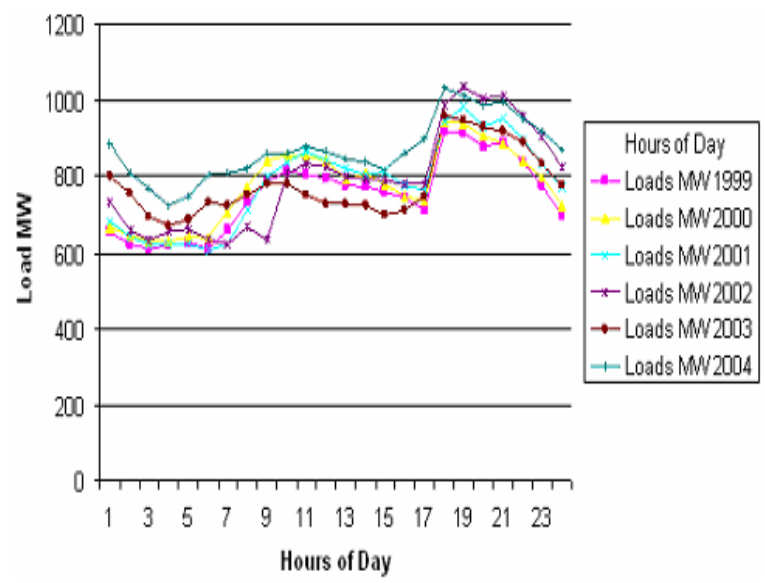

Fig. 7: System consumption at Al. Adha Eid from 1999-2004

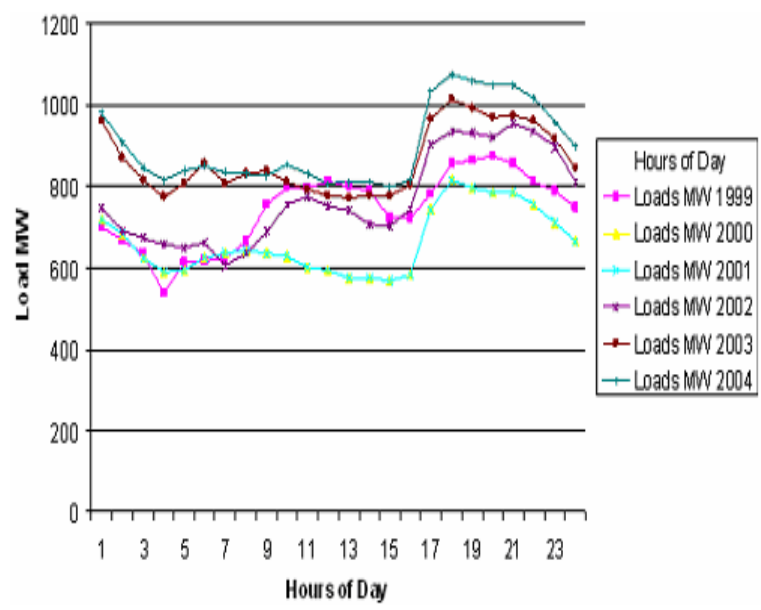

Fig. 8: System consumption at Al. Fiter Eid from 1999- 2004

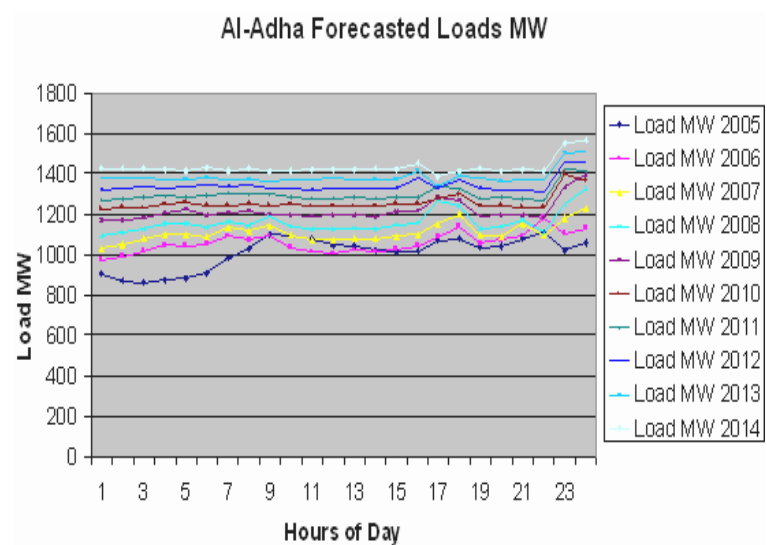

Fig. 9: Al. Adha forecasted load from 2005- 2014 


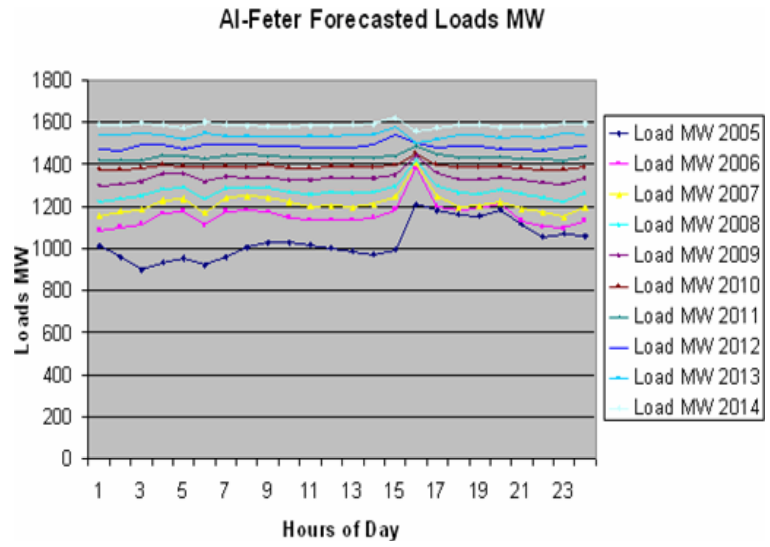

Fig. 10: Al. iter forecasted load from 2005- 2014

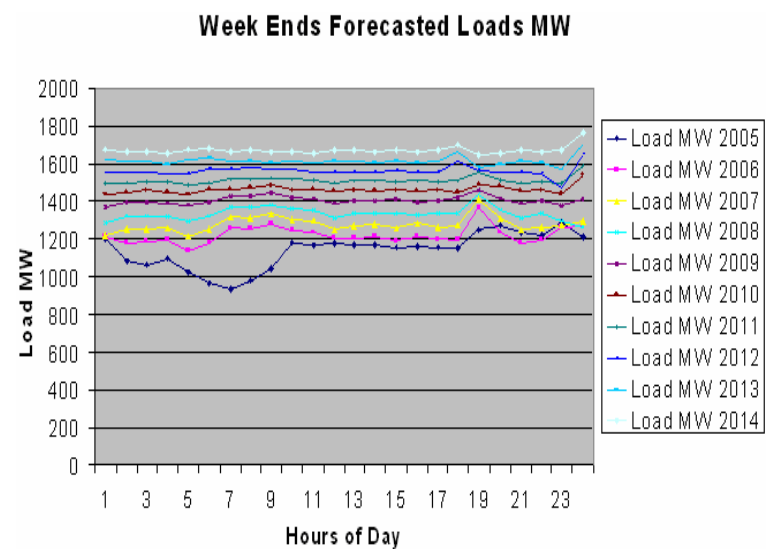

Fig. 11: Weekend forecasted load from 2005- 2014

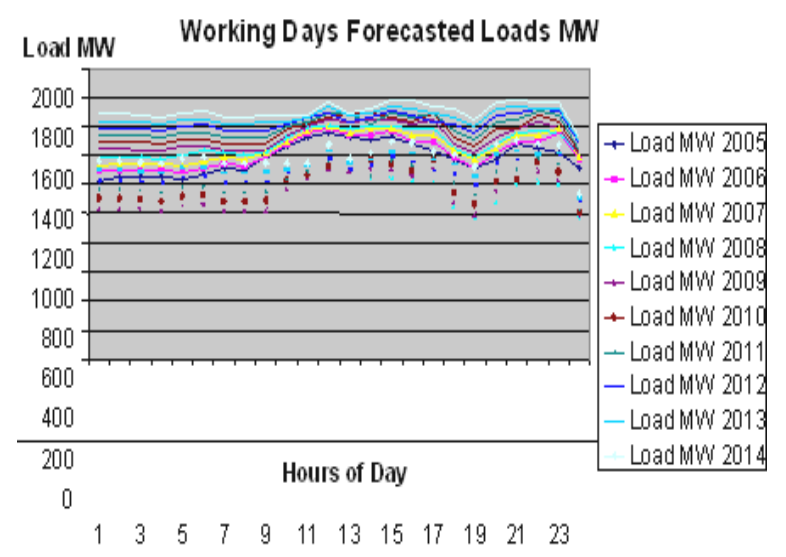

Fig. 12: Working days forecasted load from 2005- 2014

The results of the medium-term daily load forecast show slight changes in the peak load of the Eid holidays (around $1600 \mathrm{MW}$ ) for the ten years ahead, while there are a significant increase in the weekends and working days peak load (around $1800 \mathrm{MW}$ and $2000 \mathrm{MW}$ respectively). These results are depicted in Fig. 11 and 12.

\section{CONCLUSIONS}

In this study, the methods for forecasting electric loads on a power system were discussed. Emphasis was placed on short-term load forecasting which is important for real time operation and control of power systems. Deterministic, probabilistic and stochastic forecasting models were examined. The performance of these models is dependent on the characteristics of electric loads and are based on the assumption that electric load patterns are basically invariant with time ${ }^{[5]}$.

The load forecasting methodology adopted in this study proved its accuracy during the last six years of implementation.

The peak demand occurrence at day or night in the future will depend on the change of the current Tariff structures and the definition of the peak period.

Electric load forecasting plays a central role in the operation and planning of electric power. The countrywide energy estimation, the planning for new plants, the routine of maintaining and scheduling daily electrical generation are all dependent on accurate load forecasting in the future. So it is important to have a forecasting model in each power station with feed back to the central control room in order to run the system with higher efficiency and better reliability.

\section{REFERENCES}

1. National Electric Power Company Report, 2005. Jordan.

2. National Electric Power Company, 2005. "Annual Report 1990-2004", National Electric Power Company, Jordan.

3. BAJAY, S.V., 1983. "Long- Term Load Forecasting Models: A review of methodologies", Electric Power System Research.

4. Stoll, H.G., 1989. "Least-Cost Electric Utility Planning", John Wiley and Sons, USA.

5. Li, F. and Q. Jiaju, 2006. "Electrical Load Forecasting Based on Load Patterns", Power System Technology, 29: 23-26. 